\section{Mannich Reaction as a New Route to Pyridine- Based Polyaminocarboxylic Ligands}

Silvio Aime, Camilla Cavallotti, Eliana Gianolio, Giovanni B. Giovenzana," Giovanni Palmisano* and Massimo Sisti

\section{Supporting Information}

All chemicals and solvents were purchased from Aldrich, except for 3,5-dihydroxypyridine, obtained from Specs\&BioSpecs (NL) and used as received. Melting points were determined in open capillary tubes on a Büchi 510 apparatus and have not been corrected. NMR spectra were registered on Bruker AC200 or Jeol Eclipse ECP300 spectrometer. Mass spectra were obtained on a Bruker Esquire 3000 Plus spectrometer (ESI-mode). Elemental analyses were performed with a Perkin Elmer 240 apparatus.

\section{Compound 8}

In a $25 \mathrm{~mL}$ round-bottomed flask, a mixture of 3,5dihydroxypyridine $(0.300 \mathrm{~g}, \quad 2.7 \mathrm{mmol})$, diethyl iminodiacetate $(1.135 \mathrm{~g}, 6.0 \mathrm{mmol})$ and paraformaldehyde $(0.180 \mathrm{~g}, 6.0 \mathrm{mmol})$ in EtOH $(10.0 \mathrm{~mL})$ was refluxed for 18 h. Paraformaldehyde $(3 \times 0.030 \mathrm{~g}, 3.0 \mathrm{mmol})$ was periodically added to drive the reaction to completion (monitored by TLC, $\mathrm{SiO}_{2}$ plates, elution with dichloromethane/methanol/conc. aq. ammonia 98/2/0.2, spot visualized with Dragendorff spray-reagent). The solvent was removed and replaced with dichloromethane $(5.0 \mathrm{~mL})$; to this solution di-t-butyl dicarbonate $\left(\mathrm{BOC}_{2} \mathrm{O}\right.$, $0.600 \mathrm{~g}, 2.8 \mathrm{mmol}$ ) was added to convert unreacted diethyl iminodiacetate to N-BOC derivative, more easily separated by chromatography. The solution was stirred for $24 \mathrm{~h}$, then evaporated to dryness. The residue was subjected to column chromatography $\left(\mathrm{Al}_{2} \mathrm{O}_{3}\right.$, Brockmann activity III, elution with hexane/EtOAc 6/4), obtaining analytically pure $8(799 \mathrm{mg}, 58 \%)$ as a colorless oil. ${ }^{1} \mathrm{H}-$ NMR (CDCl $)$ : $1.29(\mathrm{t}, 7.1 \mathrm{~Hz}, 12 \mathrm{H}), 3.52(\mathrm{~s}, 8 \mathrm{H}), 4.04$ (s, 4H), $4.22(\mathrm{q}, 7.1 \mathrm{~Hz}, 8 \mathrm{H}), 6.76(\mathrm{~s}, 1 \mathrm{H}) .{ }^{13} \mathrm{C}\left\{{ }^{1} \mathrm{H}\right\}-\mathrm{NMR}$ $\left(\mathrm{CDCl}_{3}\right) 14.1\left(\mathrm{CH}_{3}\right), 54.2\left(\mathrm{CH}_{2}\right), 58.4\left(\mathrm{CH}_{2}\right), 61.0\left(\mathrm{CH}_{2}\right)$, $111.5(\mathrm{CH}), 132.4(\mathrm{C}), 154.7(\mathrm{C}), 170.6(\mathrm{C})$. ESI-MS: $\mathrm{m} / \mathrm{z}$ $=552\left(\mathrm{MK}^{+}\right), 536\left(\mathrm{MNa}^{+}\right), 514\left(\mathrm{MH}^{+}\right), 347,325$.

\section{Compound 1}

Compound $8(0.480 \mathrm{~g}, 0.93 \mathrm{mmol})$ was dissolved in $6 \mathrm{~N}$ $\mathrm{HCl}(10 \mathrm{~mL})$ and the solution refluxed for $8 \mathrm{~h}$. The solvent was evaporated and the residue was crystallized from ethanol/water, obtaining ligand $\mathbf{1}(381 \mathrm{mg}, 90 \%)$ as colorless crystals. M.p. $162-165^{\circ} \mathrm{C}(\mathrm{dec})\left(\mathrm{EtOH} / \mathrm{H}_{2} \mathrm{O}\right)$.

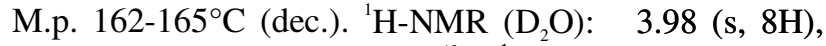
$4.43(\mathrm{~s}, 4 \mathrm{H}), 6.76(\mathrm{~s}, 1 \mathrm{H}) .{ }^{13} \mathrm{C}\left\{{ }^{1} \mathrm{H}\right\}$-NMR $\left(\mathrm{D}_{2} \mathrm{O}\right) 47.8$ $\left(\mathrm{CH}_{2}\right), 56.1\left(\mathrm{CH}_{2}\right), 118.3(\mathrm{CH}), 133.9(\mathrm{C}), 153.8(\mathrm{C})$, 169.4 (C). ESI-MS: $m / z=402\left(\mathrm{MH}^{+}\right.$, calc. for $\mathrm{C}_{15} \mathrm{H}_{19} \mathrm{~N}_{3} \mathrm{O}_{10}$ : 401) Calc. for $\mathrm{C}_{15} \mathrm{H}_{19} \mathrm{~N}_{3} \mathrm{O}_{10} \cdot \mathrm{HCl} \cdot \mathrm{H}_{2} \mathrm{O}$
(455.81): C 39.53, H 4.87, N 9.22; found: C 39.41, H $5.01, \mathrm{~N} 9.20$.

\section{Compound 9}

In a $50 \mathrm{~mL}$ round-bottomed flask, 3,5dihydroxypyridine $(0.300 \mathrm{~g}, 2.7 \mathrm{mmol})$ was dissolved in $\mathrm{MeOH}$ (15 mL). 1,4,7-Triazaheptane-1,4,7-triacetic acid tri-t-butyl ester $5(1.200 \mathrm{~g}, 2.7 \mathrm{mmol})$, tartaric acid $(0.405$ $\mathrm{g}, 2.7 \mathrm{mmol})$ and paraformaldehyde $(0.162 \mathrm{~g}, 5.4 \mathrm{mmol})$ were added and the mixture refluxed for $6 \mathrm{~h}$. After cooling, the mixture was evaporated to dryness and the residue redissolved in saturated aqueous sodium bicarbonate $(10 \mathrm{~mL})$. The suspension was extracted with dichloromethane $(3 \times 10 \mathrm{~mL})$ and the organic layers collected, dried over sodium sulphate, filtered and evaporated in vacuo. The solid residue was crystallized from EtOAc to provide compound $9(0.972 \mathrm{~g}, 62 \%)$ as colorless crystals. M.p. $157-159^{\circ} \mathrm{C}$ (AcOEt). ${ }^{1} \mathrm{H}-\mathrm{NMR}$ $\left(\mathrm{CDCl}_{3}\right): \quad 1.46(\mathrm{~s}, 9 \mathrm{H}), 1.47(\mathrm{~s}, 18 \mathrm{H}), 2.76(\mathrm{~m}, 8 \mathrm{H}), 3.17$ (s, 4H), $3.30(\mathrm{~s}, 2 \mathrm{H}), 3.76(\mathrm{~s}, 4 \mathrm{H}), 6.53(\mathrm{~s}, 1 \mathrm{H}), 9.50$ (bs, 2H). ${ }^{13} \mathrm{C}\left\{{ }^{1} \mathrm{H}\right\}$-NMR $\left(\mathrm{CDCl}_{3}\right) 28.1\left(\mathrm{CH}_{3}\right), 28.2\left(\mathrm{CH}_{3}\right), 50.8$ $\left(\mathrm{CH}_{2}\right), 51.3\left(\mathrm{CH}_{2}\right), 55.0\left(\mathrm{CH}_{2}\right), 55.8\left(\mathrm{CH}_{2}\right), 59.2\left(\mathrm{CH}_{2}\right)$, 81.5 (C), 81.7 (C), $112.0(\mathrm{CH}), 133.2(\mathrm{C}), 154.3(\mathrm{C})$, 170.1 (C), 170.2 (C). ESI-MS: $m / z=603\left(\mathrm{MNa}^{+}\right), 581$ $\left(\mathrm{MH}^{+}\right)$.

\section{Compound 2}

Compound 8 ( $0.450 \mathrm{~g}, 0.78 \mathrm{mmol})$ was dissolved in a mixture of trifluoroacetic acid $(10.8 \mathrm{~mL})$ and anisole $(2.7$ $\mathrm{mL}$ ). The clear solution was stirred at room temperature for $24 \mathrm{~h}$, then evaporated in vacuo and the residue was redissolved in $\mathrm{MeOH}(2 \mathrm{~mL})$; $t$-butylmethylether $(5.0$ $\mathrm{mL}$ ) was slowly added. The precipitate formed was isolated by centrifugation and the dissolutionprecipitation procedure repeated twice. This solid was washed with $t$-butylmethylether then dried in vacuo to give ligand $2(0.302 \mathrm{~g}, 94 \%)$ as an off-white solid. M.p. 162-164 ${ }^{\circ} \mathrm{C}$ (dec.). ${ }^{1} \mathrm{H}-\mathrm{NMR}\left(\mathrm{D}_{2} \mathrm{O}\right): 3.08(\mathrm{~m}, 4 \mathrm{H}), 3.41$ $(\mathrm{m}, 4 \mathrm{H}), 3.66(\mathrm{~s}, 2 \mathrm{H}), 4.22(\mathrm{~s}, 4 \mathrm{H}), 4.70(\mathrm{~s}, 4 \mathrm{H}), 7.04(\mathrm{~s}$, $1 \mathrm{H}) .{ }^{13} \mathrm{C}\left\{{ }^{1} \mathrm{H}\right\}$-NMR $\left(\mathrm{D}_{2} \mathrm{O}\right) 51.1\left(\mathrm{CH}_{2}\right), 54.6\left(\mathrm{CH}_{2}\right), 55.7$ $\left(\mathrm{CH}_{2}\right), 55.9\left(\mathrm{CH}_{2}\right), 57.3\left(\mathrm{CH}_{2}\right), 111.8(\mathrm{CH}), 129.2(\mathrm{C})$, 151.9 (C), 169.9 (C), 175.0 (C). ESI-MS: $m / z=435$ $\left(\mathrm{MNa}^{+}\right), 413\left(\mathrm{MH}^{+}\right.$, calc. for $\left.\mathrm{C}_{17} \mathrm{H}_{24} \mathrm{~N}_{4} \mathrm{O}_{8}: 412\right)$. Calc. for $\mathrm{C}_{17} \mathrm{H}_{24} \mathrm{~N}_{4} \mathrm{O}_{8}$ (412.40): C 49.51, H 5.87, N 13.59; found: C 49.32, H 6.00, N 13.43. 